\title{
Semences paysannes en Europe : enjeux et perspectives
}

\author{
Riccardo Bocci ${ }^{1}$ \\ Véronique Chable ${ }^{2}$ \\ ${ }^{1}$ Association italienne pour I'agriculture \\ biologique (AIAB), \\ via di Casignano 25, \\ Scandicci (Florence), \\ Italie \\ $<$ r.bocci@aiab.it> \\ ${ }^{2}$ Institut national de la recherche \\ agronomique (Inra), \\ SAD Paysage, \\ 65 , rue de Saint-Brieuc, \\ 35042 Rennes, \\ France \\ <veronique.chable@rennes.inra.fr>
}

\begin{abstract}
Résumé
Le mythe du progrès technique a imposé une conception dominante de l'" amélioration des plantes " où les métiers d'agriculteur et de sélectionneur sont devenus distincts. La séparation fut renforcée par la réglementation du marché des semences (critères stricts d'inscription de variétés à un catalogue officiel et de certification des graines). Quelques pionniers, autour des paysans pratiquant une agriculture en marge de l'industrialisation, le plus souvent une agriculture biologique $(\mathrm{AB})$, proposent aujourd'hui une alternative. En Europe, au début des années 2000, ils se sont organisés en réseaux : le Réseau Semences Paysannes en France, la Red de Semillas en Espagne, et la Rete Semi Rurali en Italie. Ce sont des paysans, consommateurs, artisans, chercheurs qui réunissent leurs compétences pour ouvrer à tous les niveaux - technique, réglementaire et scientifique - pour le devenir des "semences paysannes". Ce concept englobe deux réalités: une graine, organe de reproduction de la plante et condensé de son terroir, et une variété, façonnée par l'histoire et coévoluant avec les paysans. Des chercheurs accompagnent leur évolution avec le développement de projets de sélection participative qui stimulent à nouveau la biodiversité cultivée, par la création de variétés paysannes notamment pour l'AB, en redéployant les variétés anciennes stockées dans les centres de ressources génétiques ou chez des collectionneurs. Le cadre réglementaire a commencé à évoluer avec la prise en considération des variétés de conservation en Europe. Un projet européen, Farm Seed Opportunities est en cours pour apporter un support scientifique à la proposition de scénarios réglementaires en tenant compte des diversités nationales. Les débats du Nord offrent désormais différents modèles de "systèmes semenciers ". Il s'agit maintenant d'élargir le débat en favorisant les échanges des savoirs et connaissances entre paysans du Nord et du Sud, pour préserver la biodiversité agricole et stimuler l'innovation en milieu rural.
\end{abstract}

Mots clés : agrobiodiversité, approche participative, innovation, organisation paysanne, production de semences.

Thèmes : méthodes et outils, productions végétales, ressources naturelles et environnement.

\section{Abstract \\ Peasant seeds in Europe: Stakes and prospects}

The myth of technological progress in agriculture and then modern plant breeding, have resulted in a separation of farming from breeding activities. Seed laws as well have contributed to this end (e.g. by imposing strict rules for the inscription of varieties to the official catalogue). Nowadays, some pioneers, among farmers refusing industrialised agriculture and more often practicing organic agriculture, are proposing a different option. In Europe, since the beginning of this century, they have been organising themselves in networks: the Reseau Semences Paysannes in France, the Red de Semillas in Spain and the Rete Semi Rurali in Italy. Their members are farmers, consumers and scientists working together in order to reconsider the scientific, technical and legal aspects of seed production. These new varieties are designated "peasants' varieties", a concept that encompasses two main aspects: the seed, the reproductive part of the plant linked to its "terroir" and the variety, shaped by the history and coevolved with farmers. Scientists working with these networks are developing participatory plant breeding projects, which aim to broaden agrobiodiversity by creating new peasants' varieties. The basis of these projects comes from old local varieties and landraces conserved in seed banks. In Europe, the legal framework has evolved since the establishment of "conservation varieties" by the directive 98/95. The European research project Farm Seed Opportunities is on going to 
support new seed policies considering also the different national contexts. The debate in Europe now offers a range of seed systems models. It is time to enlarge this debate to southern countries through the sharing of knowledge between farmers of the North and the South, aiming at conserving agrobiodiversity and promoting rural innovation.

Key words: agrobiodiversity, farmers organizations, innovation, participatory approaches, seed production.

Subjects: natural resources and environnement, tools and methods, vegetal productions.

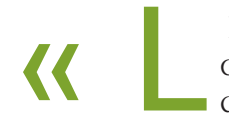

La semence est la graine que l'on sème ", d'après le dictionnaire Larousse. C'est une notion complexe qui comprend la variété et la graine. Chacun de ces deux éléments renvoie à des processus de création et de production. "On récolte ce que l'on sème ", dit le bon sens populaire. La semence résume en elle-même des choix agronomique, économique et éthique. Le mythe du progrès technique a imposé une conception dominante de l'" amélioration des plantes " accompagnant une industrialisation de l'agriculture et la séparation des métiers où les intérêts de l'agriculteur ne sont pas nécessairement ceux du sélectionneur semencier. Une poignée de pionniers propose aujourd'hui une alternative à ces choix imposés au XIX ${ }^{\mathrm{e}}$ siècle. Ces pionniers sont des citoyens, paysans, consommateurs, commerçants, chercheurs... qui réunissent leurs compétences pour se donner les moyens de travailler avec des semences conçues pour une agriculture donnant aux êtres vivants, dont les plantes, une autre dimension que celle de biens matériels et mercantiles. Le concept de "semences paysannes " englobe donc une graine, organe de reproduction de la plante et condensé matériel de son terroir et une variété, façonnée par l'histoire et coévoluant avec les paysans. L'agriculteur qui se reconnaît "paysan" donne à sa mission une attache forte au terroir, qualifié par son sol, son climat, ses hommes et leur histoire. Il ne veut plus être un "exploitant agricole". Des acteurs du développement des "semences paysannes" témoignent de l'émergence du concept en Europe, de l'organisation en réseau des mouvements nationaux, de l'évolution réglementaire nécessaire à une reconnaissance légale de leur existence. Enfin, ils souhaitent associer leur savoirfaire et leurs questions à ceux des paysans des pays du Sud qui s'interrogent et organisent le devenir de leur patrimoine végétal.

\section{Contexte des semences en Europe et émergence \\ des " semences}

\section{paysannes ॥}

Le marché des semences (production, vente et échange) est très encadré en Europe. Les normes de qualité et la propriété intellectuelle en font des produits commerciaux standardisés. L'espace légal et commercial pour la biodiversité agricole basée sur des variétés capables d'évoluer et de s'adapter à leur environnement est de plus en plus étroit (Velvée, 1993; Fowler et Mooney, 1991). La dépendance des agriculteurs vis-à-vis des semenciers est de plus en plus marquée. Néanmoins, en Europe la part des semences reproduites à la ferme (tableau 1) reste non négligeable et constitue pour l'industrie semencière un manque à gagner (Le Buanec, 2005; Grain, 2007), avec à la fois des semences fermières (variétés protégées reproduites à la ferme) et des semences illégales (variétés paysannes).

En outre, la politique agricole commune a accentué le mouvement de "modernisation" des systèmes agraires européens,

\section{Tableau 1. Origine de variétés de céréales, légumineuses et pomme de terre en Europe (Le Buanec, 2005, modifié).}

Table 1. The source of cereals, leguminous plants and potatoes in Europe (Le Buanec, 2005, modified).

\begin{tabular}{|c|c|c|c|c|}
\hline \multirow[t]{2}{*}{ Pays } & \multirow[t]{2}{*}{ Culture } & \multicolumn{3}{|c|}{ Semences } \\
\hline & & certifiées & fermières & illégales \\
\hline France & Blé & 58 & 42 & \\
\hline \multirow[t]{2}{*}{ Allemagne } & Céréales & 54 & 40 & 6 \\
\hline & Pomme de terre & 44 & 56 & \\
\hline \multirow[t]{2}{*}{ Pologne } & Blé & 7 & 93 & \\
\hline & Avoine & 5 & 95 & \\
\hline \multirow[t]{4}{*}{ Angleterre } & Blé & 51 & 31 & 18 \\
\hline & Orge d'hiver & 55 & 15 & 30 \\
\hline & Orge de printemps & 66 & 14 & 20 \\
\hline & Haricot & 37 & 18 & 45 \\
\hline \multirow[t]{4}{*}{ Italie } & Blé dur & 90 & 10 & \\
\hline & Blé tendre & 70 & 30 & \\
\hline & Luzerne & 75 & 5 & 20 \\
\hline & Orge & 80 & 20 & \\
\hline \multirow[t]{2}{*}{ Hollande } & Pomme de terre & 61 & 39 & \\
\hline & Céréales & 65 & 35 & \\
\hline Finlande & $\begin{array}{l}\text { Céréales et } \\
\text { légumineux }\end{array}$ & 30 & 47 & 23 \\
\hline \multirow[t]{2}{*}{ Suède } & Céréales & 72 & 28 & \\
\hline & Pomme de terre & 35 & 65 & \\
\hline
\end{tabular}


avec l'aide de la recherche agronomique et des lois sur les semences qui ont contribué à séparer toujours plus le paysan des semences. Celles-ci sont devenues un métier, celui des semenciers (Pistorius et van Wijk, 2000).

Dans ce contexte difficile apparaît un mouvement de réappropriation du devenir des semences dans les campagnes. Les variétés conçues et diffusées par les firmes semencières ne visent que l'agriculture industrielle et ne répondent pas aux besoins des autres agricultures. Les paysans, en agriculture biologique (AB) ou en marge de la filière agro-industrielle (Deléage, 2004), voient leurs limites :

- technique, avec les caractéristiques DHS (distinction, homogénéité, stabilité) du système Upov (Union internationale pour la protection des obtentions végétales, www.upov.int), incompatibles avec les qualités écologiques nécessaires pour ces agricultures; pour les espèces concernées, notamment les grandes cultures, l'exigence de DHS est complétée par l'évaluation de la valeur technologique et agronomique (VAT) qui oriente le progrès génétique dans une seule direction définie le plus souvent sur le rendement en agriculture intensive ou des critères de qualité industrielle ;

- politique/juridique, en posant la question de l'espace réglementaire à concevoir pour que le paysan retrouve son activité ancestrale de production de ses propres graines et surtout du type de propriété intellectuelle sur les variétés ; - scientifique, avec l'introduction des biotechnologies (OGM, mais aussi fusion et multiplication cellulaire, mutagenèse...) violant les principes de l'AB, et les recommandations actuellement établies par les Plant Breeding Draft Standards élaborées par l'International Federation of Organic Agricultural Mouvements ${ }^{1}$.

En Europe, partant de là, des paysans tentent de se réapproprier la base de leur activité : les semences dans toutes leurs dimensions intellectuelle (les variétés) et physique (les graines).

Le contexte réglementaire commence à évoluer pour offrir une alternative aux seules variétés issues de l'amélioration des espèces cultivées depuis 50 ans et inscrites au catalogue officiel. En 1998, l'Union européenne (UE), préoccupée par la perte de biodiversité agricole, a proposé une nouvelle directive (98/95/CE), avec

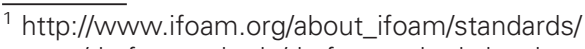
norms/draft_standards/draft_standards.html laquelle on entrevoit une possibilité de cultiver, échanger et vendre des variétés en péril d'érosion génétique, appelées "variétés de conservation ", ainsi que des variétés adaptées à l'agriculture biologique et des mélanges d'espèces et de variétés. Ces variétés présentent des caractéristiques différentes de celles qui répondent aux critères du catalogue et de l'Upov, notamment en matière d'uniformité et de stabilité. Le Plan d'action européen en faveur de la diversité biologique dans le domaine de l'agriculture a confirmé l'importance de l'existence d'une législation plus ouverte à la biodiversité (http://europa.eu/

scadplus/leg/fr/lvb/128176.htm). En effet, ce Plan, élaboré par la Commission européenne en 2001 (document COM/2001/0162 final), souligne que "la conservation et l'amélioration des ressources génétiques végétales in situ ou "à la ferme" dépendent également de la possibilité effective de ses utilisations durables et donc d'une législation permettant de commercialiser des matériaux génétiques diversifiés ". Il manque encore les décrets d'application sur les variétés de conservation. Une nouvelle directive a été proposée cette année, 9 ans après l'apparition du terme "variété de conservation". Ce délai met en évidence la difficulté de trouver un compromis sur la question avec les semenciers. De plus, le 31 mars 2004, l'UE a ratifié le Traité international sur les ressources phytogénétiques pour l'alimentation et l'agriculture (www.planttreaty.org), en ouvrant ainsi en Europe le débat sur la question des droits des paysans figurant dans l'article 9.

\section{Organisations}

\section{paysannes et recherche}

Très récemment, des réseaux paysans ont émergé pour donner une alternative à l'avenir de leurs "semences ": en France (le Réseau Semences Paysannes - RSP), en Espagne (la Red de Semillas - RdS) et en Italie (la Rete Semi Rurali). Leurs caractéristiques et leurs organisations montrent des traits communs :

- ils associent différents acteurs de la société civile concernés par la biodiversité cultivée (associations, syndicats agricoles, institutions...);

- ils sont nés au début des années 2000 et montrent une expansion rapide, mesurée par le nombre d'associations adhérentes et par le nombre d'actions menées (projets de recherche en partenariat, foire à la biodiversité, publications, formations...); - ils ont la capacité de communiquer avec le grand public et de faire partager leurs préoccupations ;

- leur travail au niveau national s'accompagne de la prise de conscience de la nécessité d'élargir le travail commun aux niveaux européen et international (Anonyme, 2005). Ils appartiennent aussi à d'autres réseaux de la société civile.

Ils se différencient d'une organisation paysanne professionnelle du fait qu'ils recrutent au-delà des paysans euxmêmes et associent tous les citoyens se sentant concernés par les semences pour le choix de leur alimentation, de leurs fibres vestimentaires, de la sauvegarde des écosystèmes, du paysage agricole...

Le Réseau Semences Paysannes en France s'est constitué en 2003 suite aux premières Rencontres Semences Paysannes "Cultivons la biodiversité dans les fermes " d'Auzeville (www.semencespaysannes.org). Il associe des associations ou organisations, et tous ceux qui œuvrent pour le développement des semences paysannes. Ainsi, les associations adhérentes représentent à la fois des groupements d'agriculteurs (syndicats, coopératives...), des artisans semenciers, d'autres structures associatives ou professionnelles (parcs naturels, collectionneurs amateurs...). Le RSP travaille à consolider l'activité et le développement des groupes de sélection, conservation, diffusion de semences paysannes. Il joue un rôle important pour stimuler l'évolution juridique et les échanges d'expériences et de ressources au niveau international, pour induire et assurer les partenariats avec la recherche publique. Il développe une stratégie de publication et de communication pour toucher un large public (publications, bulletin, site internet).

La Red de Semillas "Resembrando e Intercambiando" (www.redsemillas.info/) est une organisation espagnole technique, sociale et politique formée par des personnes et des organisations qui travaillent pour maintenir la biodiversité agricole dans les fermes des paysans et dans les assiettes des consommateurs. Ce groupe fonctionne depuis 1999 mais n'a adopté un statut légal d'association qu'en 2005. La richesse du réseau RdS réside dans la diversité des personnes et organisations qui le constituent. Dans le réseau participent des paysans et organisations agraires, des techniciens, des consommateurs, 


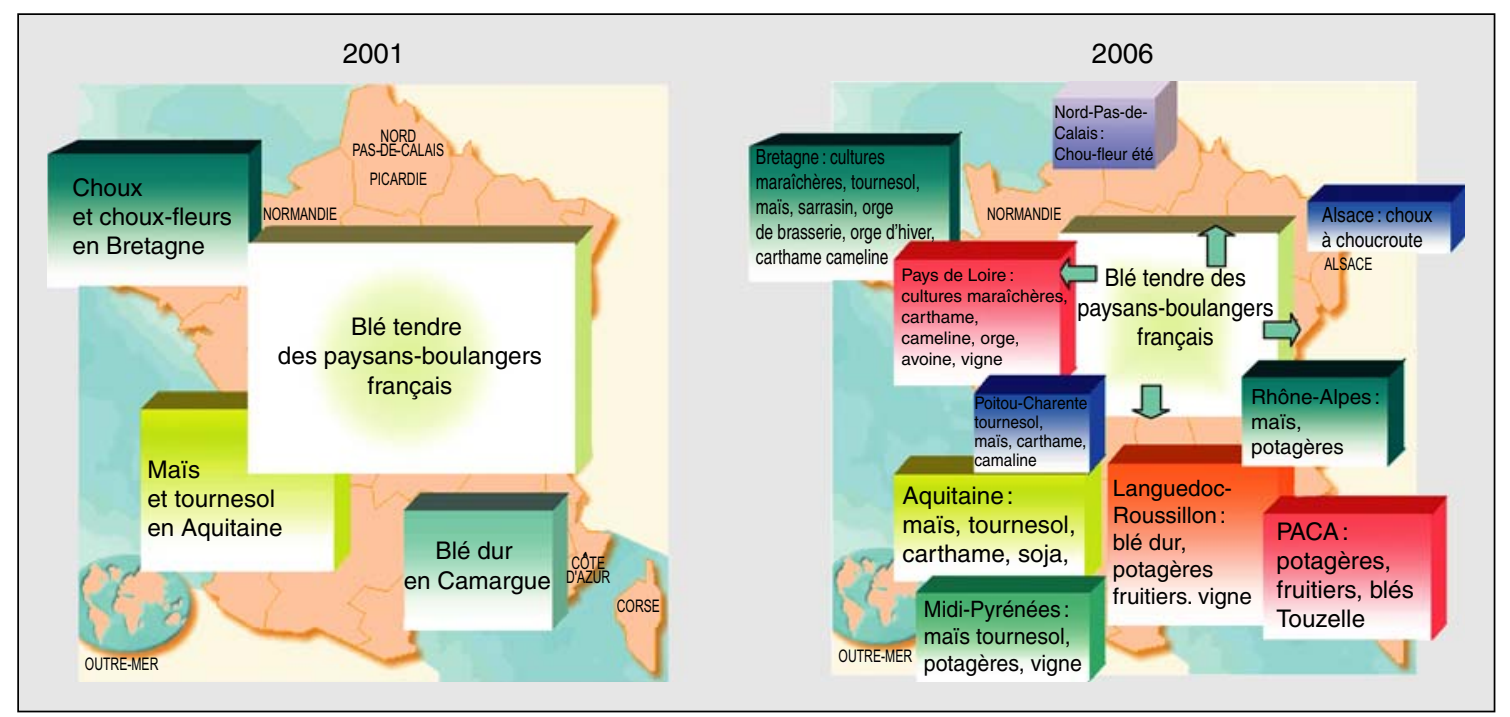

Figure 1. Espèces concernées par la sélection participative en France et évolution de leur répartition dans les régions françaises entre 2001 et 2006.

Figure 1. Plant species involved in participatory plant breeding in France and its trend between 2001 and 2006.

des groupes d'action locale et de personnes liées aux universités et à la recherche, etc.

La Rete Semi Rurali (www.semirurali.net), en Italie, est née comme groupe informel de chercheurs, paysans et agronomes en 2001 et a produit un bulletin d'information sur les activités de ses membres. Ce réseau a travaillé surtout au niveau des gouvernements régionaux qui depuis 10 ans ont promulgé des lois régionales pour la sauvegarde de la biodiversité agricole locale (Bocci et Onorati, 2006). Le travail législatif a produit un texte de lois sur les variétés de conservation qui a été approuvé par le Gouvernement en avril 2007. Cette année, le groupe informel s'est donné un statut légal sous forme d'association.

À partir de leur action initiale - préserver les semences fermières (France) ou sauvegarder les anciennes variétés (Italie et Espagne) - les objectifs et les actions des réseaux se sont élargis. Ils se sont ouverts à la recherche et à l'innovation variétale produites par les paysans eux-mêmes. Cette évolution est très marquée en France, où la recherche publique s'est aussi investie dans l'aventure paysanne, par le biais de la sélection participative (SP), notamment pour l'agriculture biologique. Les premiers projets ont commencé en 2001 par un financement public spécifique (Chable et Conseil, 2005 ; Chable et Kastler, 2006). Partant de 5 espèces en 2001, nous comptons aujourd'hui plus de 20 espèces sélectionnées aussi bien en grande culture qu'en espèces légumières (figure 1). Les réseaux paysans se sont diversifiés et connectés entre eux pour échanger les expériences et les résultats d'expérimentations. Le nombre de paysans impliqués augmente rapidement, mais aussi, après une première expérience, la plupart d'entre eux s'intéressent à plusieurs espèces en même temps. On observe une évolution des projets paysans avec la découverte de la biodiversité des espèces, les caractéristiques des variétés découvertes inspirant parfois de nouvelles opportunités de valorisation ou de techniques culturales.

Le développement des réseaux européens s'accompagne aussi de projets de

\section{Tableau 2. Participants au Projet européen Farm Seed Opportunities.}

Table 2. Participants of the EU Project Farm Seed Opportunities.

\begin{tabular}{l|l|c|c}
\hline & \multicolumn{1}{|c|}{ Nom } & Acronyme & Pays \\
\hline 1 & Institut national de la recherche agronomique & Inra & FR \\
\hline 2 & Associazione Italiana per I'Agricoltura Biologica & AIAB & IT \\
\hline 3 & Louis Bolk Instituut & $\mathrm{LBI}$ & $\mathrm{NL}$ \\
\hline 4 & Red Andaluza De Semillas & $\mathrm{RAS}$ & $\mathrm{ES}$ \\
\hline 5 & Réseau Semences paysannes & $\mathrm{RSP}$ & $\mathrm{FR}$ \\
\hline 6 & Plant Research International & $\mathrm{PRI}$ & $\mathrm{NL}$ \\
\hline 7 & $\begin{array}{l}\text { International Institute for Environment and } \\
\text { Development }\end{array}$ & $\mathrm{IIED}$ & $\mathrm{UK}$ \\
\hline 8 & Research Institute of Organic Agriculture & $\mathrm{FiBL}$ & $\mathrm{CH}$ \\
\hline 9 & Inra Transfert & $\mathrm{IT}$ & $\mathrm{FR}$ \\
\hline 10 & Wageningen University & $\mathrm{WU}$ & $\mathrm{NL}$ \\
\hline 11 & $\begin{array}{l}\text { Istituto di genetica e sperimentazione agraria } \\
\text { Nazareno Strampelli }\end{array}$ & $\mathrm{IGSA}$ & $\mathrm{IT}$ \\
\hline 12 & Stichting Dienst Landbouwkundig Onderzoek & $\mathrm{DLO}$ & $\mathrm{NL}$ \\
\hline
\end{tabular}

collaboration impliquant leur propre réseau mais s'étendant aussi à la zone méditerranéenne. Leur dynamisme s'est récemment concrétisé par l'élaboration d'un projet de recherche européen du sixième Programme-cadre Farm Seed Opportunities (Opportunities for farm seed conservation, breeding and production -www.farmseed.net) avec la participation de centres de recherche européens (tableau 2). Dans le cadre de ce programme de 3 ans, avec les acteurs concernés, les partenaires se sont fixés pour objectifs de fournir à la communauté scientifique et politique un état des lieux du marché des variétés de conservation et des variétés paysannes, de définir

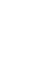

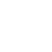


les atouts et handicaps de ces variétés par une expérimentation conduite sur plusieurs pays, de proposer des scénarios réglementaires tenant compte des aspects étudiés. Ce projet s'appuie sur les connaissances acquises au cours des expériences de SP et vise à consolider scientifiquement les acquis sur les multiples expériences. Il essaiera de définir la variété paysanne dans ses aspects fondamentaux sur 4 espèces (blé, mais, épinard et haricot) : capacité d'évolution et d'adaptation aux terroirs. Il cherchera à déterminer les atouts et les limites en matière de marché actuel et potentiel, notamment en prenant en compte les aspects de qualité des semences.

La sélection participative mise en ouvre pour le développement des variétés paysannes représente à la fois la valorisation d'un héritage des communautés paysannes et une création de nouvelles variétés. L'héritage du passé dépasse largement le cadre des variétés locales puisqu'à la base la SP puise dans les centres de ressources génétiques (RG) de toute la planète. L'aspect innovant de cette sélection paysanne n'est en fait qu'un simple retour à une tradition où la sélection et la production, mais aussi les échanges, de semences appartenaient aux paysans et faisaient partie de leur activité agricole. Le rôle du chercheur est d'accompagner le paysan pour l'aider à se réapproprier la semence. Le travail commence par la recherche des RG compatibles avec les principes de l'AB, en contrôlant les techniques de sélection des variétés expérimentées utilisées dans le passé pour éviter toute introduction de biotechnologies. Les savoir-faire paysans étant aussi souvent oubliés dans les pays du Nord, il s'agit de retrouver ensemble des pratiques compatibles avec une activité de production et une bonne qualité des semences. Ainsi, la recherche quitte le laboratoire et revient chez les paysans dans un esprit d'échanges. Le rôle du chercheur est aussi bien souvent de rapprocher les différentes expériences paysannes pour les enrichir mutuellement (Almekinders et Hardon, 2007).

\section{Savoir-faire paysan et innovation scientifique}

Ces mouvements des paysans en Europe posent aussi un questionnement politi- que. Affirmer leur capacité à sélectionner et maintenir des variétés, à produire et multiplier des semences (Cleveland et Soleri, 2002), remet en cause directement les prérogatives de la communauté scientifique qui s'est approprié l'innovation en agriculture. Le "savoir paysan " fait émerger une approche scientifique plus holistique et locale basée sur une observation rigoureuse des processus naturels, en refusant les apports des biotechnologies, vues comme un facteur de déstabilisation des systèmes d'adaptation et d'évolution des systèmes écologiques (Chable et Berthellot, 2006). Les droits et le savoir-faire paysans induisent ainsi une véritable innovation scientifique où le vivant est appréhendé dans sa globalité.

La recherche agricole est relocalisée en passant d'un modèle centralisé à un modèle décentralisé et participatif (Ceccarelli, 1994). Dans les pays du Sud, il est désormais évident que le système de l'amélioration végétale globale amené par plusieurs centres de recherche agricole internationale du Consultative Group on International Agricultural Research (CGIAR) ne suffit pas et surtout ne marche pas au dehors de l'agriculture industrielle; il a généralement favorisé des caractères de rendements et de valorisation d'intrants pour une utilisation la plus large possible, oubliant la diversité des conditions des systèmes agricoles locaux (Bellon et Morris, 2002). Le système d'inscription des variétés avec les critères VAT oriente aussi largement la sélection vers des variétés répondant à l'agriculture industrielle dans les pays du Nord.

C'est seulement depuis peu que des scientifiques redécouvrent l'intérêt d'une vision holistique de l'environnement où la problématique de la production agricole doit rejoindre celle de l'environnement (Altieri, 2004 ; Banks, 2004 ; Loreau et al., 2001). En ce sens, l'agroécologie encore à considérer comme une science en devenir, base conceptuelle des agricultures biologiques et paysannes, renouvellera le discours scientifique en sortant de l'approche majoritaire, réductionniste et analytique, des sciences agronomiques en général (Altieri, 1995). Les exemples ne manquent pas de déstabilisation des milieux écologiques par les variétés répondant aux exigences de l'inscription au catalogue officiel et se voulant de plus en plus performantes sur quelques critères économiques. Nous pouvons citer la course de plus en plus rapide entre les sources de résistance aux maladies et le développement de nouveaux pathogènes comme un signe de l'accentuation d'un déséquilibre de l'écosystème, renforcée par les techniques de production industrielle (COAG, 2007). Le système de réglementation a accompagné le paradigme scientifique de la variété stable et homogène, construit par la science génétique, et favorisant la concentration des sélectionneurs dans des multinationales de la semence avec des enjeux financiers de plus en plus colossaux, réduisant la liberté et l'autonomie du paysan en matière de semence et la biodiversité disponible (Bonneuil et al., 2006). Les paysans et autres acteurs, investissant dans l'avenir des semences au sein des réseaux, ont pris conscience qu'ils touchent finalement à des valeurs fondamentales de la société d'aujourd'hui. La réflexion s'est ainsi étendue à la place des sciences du vivant qui formatent les rapports de l'homme à la plante. Ce débat est d'autant plus crucial dans les pays qui ont encore une forte population agricole afin de préserver le patrimoine culturel et végétal encore existant. Pour les autres comme en Europe, les paysans ont la responsabilité de recréer une diversité variétale pour revitaliser une agriculture de terroir.

Partant des semences, on comprend bien vite qu'en réalité on traite de l'innovation en milieu rural : qui la produit et comment? L'innovation paysanne et même la recherche participative aboutissent à un système collectif et prennent une dimension communautaire. L'échange par la circulation des savoirs et des semences est la base de la création d'innovation (Brush, 2004). Les dispositions actuelles de protection de la propriété intellectuelle ne prennent pas en compte ce processus puisqu'elles reposent sur le concept de la propriété individuelle. Au sein d'une communauté (formelle ou informelle) dont le lien est territorial (principalement dans les pays du Sud) ou éthique (la bio des pays du Nord), les variétés sont à tous, "la semence est un don ", mais on s'organise pour gérer ensemble les graines qui représentent un travail qui doit recevoir une rétribution au sein du groupe. Dans le cas des variétés paysannes, le système de valeurs sera inversé pour protéger et favoriser l'innovation : il faudra parler des droits collectifs (Onorati, 2005; Salazar et al., 2006) et passer du concept de la propriété à celui de la reconnaissance et de la protection vis-à-vis de l'extérieur de la communauté. En effet, les droits des paysans dans le 
traité FAO sont en train d'être établis sur la base de cette réflexion (Andersen, 2006).

\section{Conclusion}

Toutes les discussions en matière de semences en Europe deviendront-elles source de réflexion pour les pays du Sud, et plus particulièrement d'Afrique?

Et inversement, toutes les études et les méthodologies sur les systèmes semenciers des paysans du Sud peuvent-elles de même être transposées au Nord?

L'agriculture biologique et/ou paysanne française et encore plus italienne possède des traits communs avec des systèmes agraires du Sud (grande variabilité agroécologique et socio-économique, fermes de petites dimensions avec une diversité d'espèces, pas d'intrants chimiques, cultures à usage multiple, préférence pour une économie locale). Les débats du Nord offrent désormais différents modèles de "systèmes semenciers ", assurant une adaptation entre les caractéristiques culturelles et sociales d'une communauté paysanne et ses techniques agricoles. Le temps est venu d'élargir le débat en favorisant les échanges des savoirs et connaissances entre paysans du Nord et du Sud, pour préserver la biodiversité agricole et stimuler l'innovation en milieu rural. Les enjeux de l'information entre les communautés paysannes sont d'autant plus importants pour l'Afrique de l'Ouest que celle-ci est en train de développer sa politique sur les semences et que son choix ne semblait limité jusqu'alors qu'aux seuls systèmes Upov ou brevet (Tripp et al., 2007). Élargir le débat entre scientifiques, paysans du Nord et du Sud, et industries semencières, est fondamen- tal pour mettre l'accent sur la légitimité de la pluralité des approches.

\section{Références}

Anonyme. Libérons la diversité. Droits des paysans et semences: les enjeux pour l'Europe. Compte rendu du Séminaire euro-

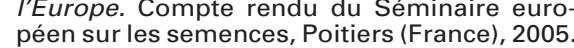

Almekinders C, Hardon J. Bringing farmers back into breeding. Agromisa Special 5. Wageningen : Agromisa, 2007.

Altieri MA. Linking ecologists and traditional farmers in the search for sustainable agriculture. Front Ecol Environ 2004 ; 2 : 35-42.

Altieri MA. Agroecology: the science of sus tainable agriculture. Boulder (Colorado) : Westview Press, 1995.

Andersen R. Governing agrobiodiversity: the emerging tragedy of the anti-commons in the South. 47th Annual Convention of the International Studies Association, San Diego (ÉtatsUnis), 2006.

Banks JE. Divided culture : integrating agriculture and conservation biology. Front Ecol Environ $2004 ; 2$ : 537-45.

Bellon M, Morris M. Linking global and local approaches to agricultural technology development : the role of participatory plant breepment : the role of participatory plant bree-
ding research in the CGIAR. CIMMYT Econoding research in the CGIAR. CIMMYT Econo-
mic working paper. Mexico: Centre international pour l'amélioration du maïs et du blé (CIMMYT), 2002.

Bocci R, Onorati A. Législation européenne pour les variétés résultant des programmes de sélection participative : état des lieux dans les régions italiennes. Proceedings of the ECO-PB régions italiennes. Proceedings of the ECO-PB
Workshop "Participatory Plant Breeding: relevance for organic agriculture? ", 2006

Bonneuil C, Demeulenaere E, Thomas F Joly PB, Allaire G, Goldringer I. Innover autrement ? La recherche face à l'avènement d'un nouveau régime de production et de régulation des savoirs en génétique végétale. Dos sier de I'Environnement de I'INRA 2006(30): sier de
$29-51$.

Brush S. Farmers' Bounty, locating crop diver sity in the contemporary world. New Haven London : Yale University Press, 2004.

Ceccarelli S. Specific adaptation and breeding for marginal environments. Euphytica 1994 $77:$ 205-19.
Cleveland DA, Soleri D. Farmers, scientists and plant breeding: integrating knowledge and practice. New York: CABI Publishing, 2002.

Chable V, Berthellot JF. La sélection participative en France : Présentation des expériences en cours pour les agricultures biologiques et paysannes. Les dossiers de l'Environnement de I'INRA 2006 ; (30) : 129-38.

Chable V, Conseil M. Variétés et semences de choux et choux-fleurs pour l'agriculture biologique : de l'évaluation des ressources génétiques vers l'organisation d'une filière semences. Séminaire sur les recherches en agriculture biologique INRA-ACTA, Draveil, 20-21 novembre 2005.

Chable V, Kastler G. Maintien, redécouverte et création de la diversité cultivée pour l'AB. Alter Agri 2006 ; (juillet-août) : 13-7.

Committee on Agriculture (COAG). Environment and Agriculture. Rome : Food and Agriculture Organisation (FAO), 2007.

Deléage E. Paysans de la parcelle à la planète. Paris : Edition Syllepse, 2004.

Fowler C, Mooney P. Shattering: food, politics, and the loss of genetic diversity. Tucson : University of Arizona Press, 1991.

Grain. The end of farm-saved seed? Industry's wish list for the next revision of UPOV. Grain Briefing 2007 ; (February) : 1-12.

Loreau M, Naeem S, Inchausti $\mathrm{P}$, et al. Biodiversity and ecosystem functioning: current knowledge and future challenges. Science $2001 ; 294$ : 804-8.

Le Buanec B. Enforcement of plant breeders' rights. Meeting on Enforcement of Plant Breerights. Meeting on Enforcement of Plant Breeders' Rights, UPOV/ENFORCEMENT/05/3,
Geneva, 25 October 2005 (non publié sur le site de I'Upov, mais consultable sur: www.grain.org/blr_files/ueisf.pdf).

Tripp R, Louwaars NP, Eaton D. Plant variety protection in developing countries. A report from the field. Food Policy 2007 ; 32 : 354-71.

Onorati A. Collective rights over farmers' seeds in Italy. Seedling 2005 ; July : 17-21.

Pistorius R, van Wijk J. The Exploitation of Plant Genetic Information. London: Oxford University Press, 2000.

Salazar R, Louwaars NP, Visser B. On protecting farmers' new varieties: new approaches to rights on collective innovations in plant genetic resources In: CAPRi Working Paper 45. Washington (DC) : 45. Washington (DC) : Institut international de recherche sur les politiques alimentaires (IFPRI), 2006.

Velvée R. The decline of diversity in European agriculture. Ecologist $1993 ; 23: 64-9$. 